\title{
El término Educación Física en la postmodernidad: contribución de algunas perspectivas fenomenológicas \\ The term Physical Education in postmodernity: contribution of some phenomenological perspectives \\ Felipe Nicolás Mujica Johnson \\ Universidad Católica de Temuco (Chile)
}

\begin{abstract}
Resumen: La modernidad nos dejó como legado el descubrimiento de la subjetividad y una concepción mecanicista del cuerpo humano. Así, diferentes filósofos de la escuela fenomenológica han conservado el patrimonio de la subjetividad y han trabajado para superar el dualismo cartesiano. Sin embargo, los avances filosóficos por múltiples razones no siempre fecundan en el campo pedagógico, de modo que es fundamental promover una constante reflexión filosófica en el contexto de la Educación Física. Por esa razón, se ha desarrollado este ensayo que tiene por objetivo analizar la perspectiva del cuerpo humano en la corriente fenomenológica alemana del siglo XX, en concreto, en las ideas de Edmund Husserl, Max Scheler y Edith Stein. Por medio del estudio de sus diferentes obras, se puede mencionar que hay un concepto clave en sus ideas, que es el del cuerpo vivo. Este cuerpo expresaría el espíritu o el sujeto encarnado, que experimenta la realidad a través de su ser psicofísico. Entonces, el ser humano tendría en todas sus experiencias un cuerpo vivo pero, a su vez, también físico o material. Por consiguiente, de este análisis se concluye que para superar la concepción mecanicista del cuerpo en la Educación Física, no es necesario cambiar el nombre a dicha disciplina pedagógica, sino comprender que la materia del ser humano es inseparable de su subjetividad.
\end{abstract}

Palabras clave: corporalidad, subjetividad, educación formal, afectividad, racionalismo.

\begin{abstract}
Modernity left us the discovery of subjectivity and a mechanistic conception of the human body as a legacy. Thus, different philosophers of the phenomenological school have preserved the patrimony of subjectivity and have worked to overcome the Cartesian dualism. However, philosophical advances do not always fertilize the pedagogical field for many reasons, so it is essential to promote a constant philosophical reflection in the context of Physical Education. For this reason, this essay has been developed with the aim to analyze the perspective of the human body in the German phenomenological stream of the 20th century, in particular, in the ideas of Edmund Husserl, Max Scheler, and Edith Stein. Through the study of their different works, it can be mentioned that there is a key concept in their ideas, i.e. the living body. This body would express the spirit or the embodied subject, who experiences reality through his/her psychophysical being. Then, the human being would have, in all his/her experiences, a living body, but also physical or material. Therefore, from this analysis it is concluded that to overcome the mechanistic conception of the body in Physical Education, it is not necessary to change the name of said pedagogical discipline, but to understand that the human being's matter is inseparable from its subjectivity.
\end{abstract}

Key Words: corporality, subjectivity, formal education, affectivity, rationalism.

\section{Introducción}

La educación formal en occidente ha estado y sigue estando marcada por el dualismo cuerpo y alma de la tradición platónico-cartesiana (Gallo, 2007). Y la influencia de dicha tradición filosófica a la pedagogía ha tenido consecuencias negativas para el desarrollo de una educación integral del ser humano, ya que ha devaluado el cuerpo y las emociones del ser humano. Estos efectos han sido influenciados en primera instancia por algunas de las ideas que proclamó Platón (2002) y, específicamente, la existencia en las personas de un alma que sería independiente del cuerpo humano. El dualismo platónico queda resumido en que el cuerpo es «el sujeto de las emociones, y el responsable de la percepción sensorial; mientras que el alma es presentada como el sujeto de la facultad privilegiada del entendimiento» (Echenique, 2018, p. 56). Y, en segunda instancia, fueron influenciados por las nociones que posteriormente estableció Descartes (Baigorri, Cifuentes, Ortega, Pichel y Trapiello, 1995), resumidas en que el alma humana además de ser independiente, se reduce a la conciencia o racionalidad. De esta forma, aquel filósofo francés convirtió al cuerpo en un obje-

Fecha recepción: 15-06-19. Fecha de aceptación: 15-11-19 Felipe Nicolás Mujica Johnson fmujica@live.cl to ajeno y sumiso al alma. Precisamente, una idea que refleja la división cartesiana entre el alma racional y el cuerpo es la siguiente:

Advierto aquí, en primer lugar, que hay gran diferencia entre la mente y el cuerpo, pues el cuerpo es siempre por naturaleza divisible, mientras que la mente es absolutamente indivisible; pues cuando la considero, es decir cuando me considero a mí mismo en tanto que soy sólo una cosa pensante, no puedo distinguir partes en mí, sino que entiendo que yo soy una cosa completamente unitaria e íntegra (Descartes, 2003, p. 78).

Por otra parte, las emociones tuvieron mejor suerte que el cuerpo en el pensamiento cartesiano, ya que a diferencia de la filosofía platónica, fueron integradas al alma y se reconoció que la mente no tenía el control total sobre ellas. No obstante, su enfoque racionalista se impuso y las catalogó como un tipo de pensamiento (percepciones), pero un pensamiento de segunda categoría y confuso debido a la mediación que ejercía el cuerpo. Así, hasta el alma más débil, por medio de una buna conducción, podría lograr adiestrarlas y dirigirlas, de un modo similar a la contemporánea inteligencia emocional. En resumen, en la siguiente idea se refleja la subordinada concepción que le otorgó Descartes a las emociones:

Pueden llamarse percepciones, cuando nos servimos en general de esta palabra para designar todos los pensa- 
mientos que no son acciones del alma, o voliciones; pero no cuando sólo nos servimos de ella para designar conocimientos evidentes. Porque la experiencia enseña que los que están más alterados por sus pasiones no son los que las conocen mejor, y que éstas se hallan entre el número de percepciones que la estrecha alianza que existe entre el alma y el cuerpo hace confusas y oscuras (Descartes, 1997, p. 96).

Comprender aquella filosofía racionalista es importante porque ha influenciado muchos aspectos epistemológicos que están presentes en la educación formal. Entonces, con la finalidad de entender las consecuencias del racionalismo, a continuación se presentan algunas de ellas que tienen un carácter bastante general y transversal en el proceso pedagógico de las diferentes naciones occidentales: a) devaluación de los asuntos concretos referidos a la formación corporal y motriz (Águila y López, 2019; Almonacid, 2012; Contreras, 1998; Fernández-Balboa, 1993; Gallo, 2012; Moreno, Campos y Almonacid, 2012; Sicilia, 2012); b) devaluación de los asuntos referidos a la formación afectiva-moral (Damasio, 1996; Maturana, 2001, 2005; Mujica, 2018a; Mujica, Orellana y Luis-Pascual, 2019); y c) sobrevaloración de los asuntos referidos a la formación intelectual (Cagigal, 1979; Toro, 2017; Mujica, 2018b, 2019). En síntesis, la educación a nivel general en occidente ha despreocupado la formación humana integral y se ha decantado por una educación ultra racionalista. También, asociado a lo anterior es posible señalar otro de los efectos de esta concepción educativa hiperintelectualista, aunque también mezclado con una lógica neoliberal o mercantil de la educación, es la exagerada relevancia otorgada a las pruebas estandarizadas a nivel nacional e internacional (Apple, 2011; Díez, 2010; Freire, 1975; Murillo y Román, 2010; Nussbaum, 2016; Oliva y Gascón, 2016; Rivas, 2015; Vicente, 2010).

A pesar de estas consecuencias negativas, es innegable que Platón y Descartes son dos de los más grandes filósofos que nuestra historia recuerda, por lo que hasta el día de hoy siguen siendo fuentes inspiradoras fundamentales para el pensamiento contemporáneo. Con respecto a la importancia de Descartes, es bien expresada por Ortega y Gasset (2003), quien explica que aquel filósofo francés construye una gran muralla para finalizar la época medieval, dando inicio a la época de la modernidad. Junto con dicha división histórica, tiene el gran mérito de descubrir la conciencia o la subjetividad humana. En otras palabras, el humano «moderno se ha metido en sí, ha vuelto en sí, ha despertado de su inconsciencia cósmica, ha sacudido el sopor que le quedaba de hortaliza, de alga, de mamífero y ha tomado posición de sí mismo: se ha descubierto» (Ortega y Gasset, 2003, p. 176).

Sin embargo, existió también una resistencia de quienes estaban disconformes con el pensamiento medieval, como es normal ante los nuevos acontecimientos. Así, aquel pensamiento moderno fue catalogado por los cristianos de la época como anti-cristiano, a pesar de sus proximidades con San Agustín (Baigorri, et al., 1995; Ortega y Gasset, 2003), ya que rompe con la actitud mental tradicional medieval vertida hacia lo exterior. Esta irritación cristiana queda bien expresada por otro filósofo francés, Pascal (1967), que debido a las limitaciones que percibe en las ideas cartesianas, las cataloga de inútiles. Este rechazo, en parte, es por la subordinación de los sentimientos a la razón en las nociones cartesianas, contrarias a la lógica del corazón que Pascal defendió (Ferrater, 2002). Precisamente, dichas discrepancias filosóficas, como se verá posteriormente, fueron recogidas por la filosofía contemporánea y, en concreto, por representantes de la fenomenología.

Luego del prolongado tiempo que el pensamiento moderno se instaló en la sociedad occidental, podemos confirmar que para superar una perspectiva filosófica, es preciso asimilarla y comprenderla en profundidad para dar el siguiente paso en la escalera del conocimiento (Ortega y Gasset, 2003). Y una de las escuelas filosóficas que destaca por dar aquel paso, es la fenomenología, la cual se caracteriza por analizar la conciencia en su intencionalidad (Abbagnano, 2008). Cabe destacar, que existen múltiples interpretaciones de lo que define la posmodernidad. Y, justamente, entre ellas se puede apreciar que algunas tienen un significado más exhaustivo y delimitado que otras, de modo que serían diferentes formas para referirse a dicho cambio cultural (Harvey, 1998). Así, se considera como un periodo de rechazo de algunas concepciones en torno a la perspectiva moderna y, a su vez, una renovación de los diferentes pilares ideológicos que sostenían la modernidad. En este sentido, la posmodernidad integra múltiples corrientes filosóficas que rechazan diferentes principios de la ilustración (Wesley, 2012). De esta forma, como se mencionaba anteriormente, diferentes exponentes de la corriente fenomenológica se han esforzado por sentar las bases teóricas de variados asuntos que engloban la existencia humana. Y, específicamente, desde una lógica que, en ocasiones, se aleja, corrige y transforma los modernos postulados del pensamiento racionalista (Adrián, 2007). Precisamente, una de las concepciones modernas importantes en la genealogía de la Educación Física que, desde diferentes perspectivas filosóficas, se ha superado, es la del ser humano fragmentado en cuerpo-mente y cuerpo-alma.

Entonces, con la finalidad de poder vislumbrar ideas sobre la terminología Educación Física en una época postmoderna, el objetivo de este ensayo es analizar la interpretación del cuerpo y las emociones en algunos autores relevantes de la fenomenología, en concreto, Edmund Husserl, Max Scheler y Edith Stein. Cabe destacar que como suele suceder, los filósofos y la filósofa estudiada comparten en común diferentes ideas, pero también presentan discrepancias. No obstante, en este ensayo no se pretende enfocar la atención en aquellas diferencias, sino destacar sus aportaciones en común que nos abren senderos hacia una educación acorde los avances culturales y, por lo tanto, una resignificación del cuerpo y de las emociones para una práctica pedagógica postmoderna.

\section{El espíritu encarnado o el cuerpo vivo}

La fenomenología de Husserl, nace con un carácter antiempirista y anti-psicologista (Abbagnano, 2008), en defensa del pensamiento filosófico y las verdades que logra dilucidar. Por lo mismo, demostró un notable pensamiento filosófico que criticó el dualismo cartesiano, señalando que aquel camino induce a un pensamiento vacío, irreal y carente de interés filosófico (Husserl, 1991). Así, rechazando la idea del cuerpo humano como una extensión, manifiesta que la cons- 
ciencia se encuentra presente en un cuerpo vivo (Husserl, 1996), de modo que «el yo que filosofa y que posee una existencia consciente, el famoso Yo trascendental, no es otro que el Yo de carne y hueso que transita por las calles» (Herrera, 2010, p. 261).

Por esa razón, es necesario diferenciar el cuerpo vivo (Leib) o cuerpo humano que expresa el ser en el mundo y el cuerpo físico o cuerpo cosa (Körper) que expresaría los objetos materiales e incluso el cadáver del ser humano. Y, precisamente, el cuerpo vivo también es físico (Leibkörper), lo cual expresaría la realidad psicofísica del ser humano (Husserl, 1996). Entonces, es por medio de esta existencia corpórea que el ser humano experimenta su vida, conduce su comunicación y piensa significativamente en el mundo intersubjetivo (Herrera, 2010). Por último, cabe destacar que con respecto a la constitución de la persona humana, Husserl (1962) manifestó que se compone de una realidad material y una realidad espiritual. En consecuencia, Husserl reconoce en el ser humano un espíritu que es su principio absoluto, el cual le permite comprender el mundo circundante. No obstante, Husserl no le otorga el sentido trascendente que le otorgará su discípulo Scheler (Muñoz, 2012), el cual aprovechará su nutrido legado teórico para emprender una trascendencia de la vida terrenal.

Siguiendo las anteriores ideas de Husserl, Scheler (2001, 2005) mantiene que el ser humano vive sus experiencias por medio de un cuerpo vivo, el cual se encuentra compuesto por una dimensión psicofísica y otra espiritual. Ambas dimensiones completarían la noción de persona, pero el espíritu tendría una naturaleza distinta, de modo que fundamenta un dualismo antropológico entre el aspecto vital y el aspecto espiritual (Scheler, 2000). Asimismo, este filósofo alemán enfatiza que el dualismo cartesiano representa una falsa división de la consciencia y el cuerpo humano, introduciendo graves errores a la naturaleza humana en la sociedad occidental (Scheler, 2000). Así, las vidas física y psíquica no estarían unidas, ya que representan la misma vida manifestada de diferentes formas. Por lo tanto, sería falsa la idea de que el ser humano se mueve por consecuencia de una interacción causal de dos principios, debido a que el impulso vital en la fenomenología scheleriana corresponde a «un dato originario presente tanto en la dimensión psíquica como en la corporal de una y la misma vida» (Sánchez-Migallón, 2008, p. 345). En resumen, Scheler (2000) destaca que lo único bueno de la teoría de Descartes es la autonomía que le otorga al espíritu por sobre la naturaleza, pero es falsa la división res cogitans y res extensa, ya que la vida psicofísica se desarrolla en una totalidad y, además, no es mecánica. Cabe mencionar que las contribuciones más relevantes de Scheler tratan sobre la filosofía de los sentimientos y, en particular, su fenomenología se distingue de la de Husserl porque no se limita a indagar en las intencionalidades intelectuales, sino que principalmente se dedicó a estudiar la intencionalidad emocional (Ferrater, 2002). Esta línea fenomenológica se refleja claramente en su ética objetiva y material de los valores, donde las emociones permiten intuir el mundo axiológico. Respecto a las ideas cartesianas de las emociones, Scheler (2000) señala que es un grave error haber reducido la vida psíquica al pensamiento o la conciencia, ya que pasa por alto la autonomía de la vida impulsiva y afectiva.
Por otra parte, pero siguiendo la idea del cuerpo vivo, la filósofa Edith Stein defiende que la condición ontológica del yo, o sea del sí mismo, es una unidad psicosomática, de modo que es también constituida por el cuerpo humano (Haya, 2003). Además, Stein $(2004,2007)$ al igual que Scheler (2000, 2005), sostienen que el ser humano es en esencia un alma o un espíritu que trasciende la vida o la existencia terrenal que, a diferencia de Descartes (2003), no se reduce a la vida racional y se integra en una totalidad con su realidad corporal y mental. Así, por medio de la aprehensión del cuerpo que realiza el alma, es preciso señalar que alma y cuerpo en la vida humana forman una misma unidad (ser humano), de modo que nuevamente diferenciándose de la tradición platónica-cartesiana, rechaza la metáfora del cuerpo como cárcel del alma:

El alma no «habita» en el cuerpo como en una casa, no se lo pone y se lo quita como un vestido, y si los filósofos griegos lo denominaban «cárcel» y «tumba» del alma, con ello se estaban refiriendo a una vinculación estrecha (y dolorosa), pero en cualquier caso a una «vinculación», y con esta noción no se hace justicia a la unidad de la naturaleza. El cuerpo está por completo penetrado por el alma, de manera que no sólo la materia organizada se convierte en cuerpo penetrado de espíritu, sino que también el espíritu se convierte en espíritu materializado y organizado (Stein, 2007, p. 129).

Antes de continuar, es preciso aclarar algunas ideas en torno a la relación cuerpo-alma que plantea nuestra filosofa. En sus pensamientos, Stein (2007) acepta la unidad cuerpoalma planteada por Tomás de Aquino que, basándose en el hilemorfismo aristotélico, afirma, a diferencia de la perspectiva platónica-cartesiana, que el alma «está inescindiblemente unida al cuerpo por necesidad y en propio provecho» (Filippi, 2012, p. 54). En este sentido, la tesis tomista asume la interdependencia cuerpo-alma como una relación natural, desmarcándose de la visión que la concibe como una relación forzada y punitiva, en la que el alma es la prisionera del cuerpo (Gómez y Sastre, 2008). No obstante, Tomás de Aquino (1993) no se desmarca totalmente del dualismo platónico, ya que sostiene la supervivencia del alma a la muerte $\mathrm{y}$, en consecuencia, su separación del cuerpo. Igualmente sucede con Stein, quien sostiene que el alma humana sobrevive a la muerte y pasa a ser un espíritu puro finito. Esta relación cuerpo-alma que, en dicha filósofa, pasa de la unidad indivisible en la vida humana a un dualismo que le otorga independencia al alma (Taubenschlag, 2014) se aprecia en la siguiente cita:

Lo que las convierte en «almas» y las distingue de los «espíritus puros» es que son «centro del ser», «núcleo personal» de una naturaleza espiritual-corporal, de una unidad personal corporal-anímica. La unión con el cuerpo es esencial para el alma. Sin embargo, en la muerte hemos de ver la separación del cuerpo y el alma (Stein, 2007, p. 126).

Por medio de la aclaración sobre el trasfondo dualista en el que se enmarca la unidad cuerpo-alma identificada en el pensamiento de Stein, se evidencia al mismo tiempo su relación con el dualismo de Scheler, no obstante, como se puede apreciar en las ideas anteriores, existe una importante diferencia cualitativa entre ellos. En concreto, esta diferencia es 
que Scheler si bien plantea durante la existencia humana una unidad razón-emoción o cuerpo-mente, también plantea un dualismo entre la vida sensible y el espíritu. En cambio, Stein prescinde de dicho binomio en la realidad humana, planteando que «la percepción sensible es ya un acto espiritual porque es una expresión del espíritu, de la apertura de la persona» (Caballero, 2010, p. 42). En otras palabras, la unidad del cuerpo y el alma es total; y se refleja constantemente en los momentos que nuestras emociones afectan los órganos corporales (Stein, 2007). Por consiguiente, para la filósofa de Breslavia, denominada Copatrona de Europa, no existe «contraposición entre sensibilidad y espíritu, sino entre naturaleza y espíritu. Así, la piedra carece de espíritu; los animales sólo tienen funciones espirituales inferiores» (Caballero, 2010, p. 42). Justamente, estos antecedentes reflejan que cualquier época, sea moderna o postmoderna, no implican unanimidad de perspectivas, de modo que tanto Scheler como Stein se apoyan en referentes que precedieron o experimentaron la época moderna racionalista.

Por otro lado, se ha constatado que para Stein (2004) resultaba absurdo hablar sobre cuerpos humanos físicos u objetivos carentes de subjetividad, ya que sería negar su naturaleza humana. Aquella idea queda bien reflejada cuando nos manifiesta que es «absolutamente imposible un cuerpo vivo sin yo. Imaginar mi cuerpo vivo abandonado por el yo ya no quiere decir imaginar mi cuerpo vivo, sino un cuerpo físico que se le asemeja rasgo a rasgo, mi cadáver» (Stein, 2004, p. 65). Por consiguiente, en coincidencia con el pedagogo y filósofo Cagigal (1979), nuestra filósofa alemana se expresó a favor de la práctica de ejercicio físico en el marco de una formación integral del ser humano. Esto se debería a que el cuidado del cuerpo mejora las condiciones para el buen desarrollo de la vida espiritual, sin embargo, advierte que la formalización de dicha vida espiritual siempre será determinada por la voluntad del alma espiritual encarnada (Stein, 2007). Por esta razón, es posible apreciar personas que gozan de un cuerpo ejercitado y saludable, pero que carecen de buenas preferencias morales y, por tanto, espirituales.

En cuanto a los sentimientos experimentados por el ser humano en su cuerpo vivo, nuestra filósofa nos indica que podemos distinguir los sentimientos sensibles o corporales (cansancio, dolor, placer, etc.) y sentimientos anímicos o espirituales (emociones y estados de ánimo) (Stein, 2004). En este sentido, Stein (2007) nos señala que el alma se comunica o habla por medio del cuerpo y la expresión de sus emociones o movimientos anímicos. Asimismo, las emociones con los sentimientos sensibles no espirituales no existen en caminos paralelos, sino que se son inter-dependientes (Stein, 2004). Y, por último, es preciso comprender que para nuestra filósofa la relación alma y cuerpo es una unidad entre iguales, por lo tanto, no consiste en «una unidad de dos sustancias separadas, sino una materia formalizada vitalmente, cuya forma se manifiesta en la materia y simultáneamente se expresa interiormente en la actualidad de la vida anímica» (Stein, 2007, p. 56).

Luego de analizar estas perspectivas fenomenológicas del cuerpo vivo, se dará lugar en el siguiente apartado al análisis de una Educación Física orientada a superar el cuerpo máquina cartesiano.

\section{Educación Física en la posmodernidad}

El principal dilema que aborda este ensayo, es si el término Educación Física representa una educación que propicia la postura del cuerpo vivo. Quienes han tomado una postura contraria a dicha terminología, son Gallo (2009) y Sérgio (2006), inspirándose en la fenomenología del cuerpo de Merleau Ponty (1993). Cabe destacar, que dicho filósofo es continuador de las ideas de Husserl y por lo tanto del espíritu o sujeto encarnado. Así, dichos autores nos dicen que es fundamental extraer el concepto físico de la asignatura y reemplazarlo por otro término. De este modo, manifiestan que se propiciaría superar la concepción materialista-mecanicista (Peña, Toro, Osses, Pachón y Hernández-Mosqueira, 2018; Rodríguez y Hernández, 2018) que ha estado implícita por tanto tiempo en la Educación Física. Con respecto a dicha postura, pienso que no es algo fundamental para superar la perspectiva mecanicista y, a su vez, que no es correcto negar la dimensión física o material del ser humano. Asimismo, creo que es posible reemplazar conceptos en la asignatura, sobre todo en su terminología principal, sin embargo, es difícil encontrar un concepto perfecto que no pueda conducirnos a alguna interpretación indeseable. Incluso, si tuviésemos la intención de cambiar el nombre a la asignatura por Educación Corporal, también nos traería un problema, porque no existe ninguna asignatura formal que no eduque el cuerpo. En cambio, no todas las asignaturas formales incluyen en sus fines pedagógicos la dimensión física.

Por consiguiente, la intención de reemplazar el concepto físico por corporal podría tener un carácter más simbólico que ontológico desde la perspectiva fenomenológica del cuerpo vivo, ya que es sería un error pretender señalar que el ser humano no posee una dimensión física o fisiológica. Dicha dimensión ha sido bien fundamentada por Husserl (1962), Scheler (2000) y Stein (2004), y se refleja cuando distinguen la realidad psicofísica del ser humano. Asimismo, cuando manifiestan que el sujeto se expresa por medio de un cuerpo vivo y físico (Leibkörber). Por consiguiente, se considera que es innegable que el ser humano cuenta con una dimensión física, pero que, a su vez, no es un objeto sin vida. Precisamente, porque la dimensión física, de forma dinámica, se encuentra animada espiritualmente formando una unidad vital indivisible. Entonces, lo que sería fundamental es que los profesionales de la educación y, en concreto, de esta asignatura especializada, comprendan que la realidad física del ser humano esencialmente no puede ser tratada con independencia de la subjetividad humana.

En este sentido, en sintonía con las perspectivas filosóficas fenomenológicas que se han estudiado, se considera en concordancia con Cagigal $(1979,1984)$, que el ser humano posee en esencia una realidad espiritual y corporal que esencialmente son diferentes. Por lo tanto, se considera que es posible superar el mecanicismo con una unidad vital cuerpo y alma, sin necesidad de enmarcarse exclusivamente en una tradición antropológica monista, sino que también es posible por medio de una noción antropológica cualitativamente diferente a la cartesiana y que no se reduzca al monismo. Como, por ejemplo, la noción que Stein (2007) ha desarrollado siguiendo la línea argumentativa del hilemorfismo aristotélico y tomista pero, de igual forma, abriendo la puerta 
a un dualismo posterior a la muerte. Por lo tanto, la evolución histórica del cuerpo humano no abarca solamente concepciones dualistas y monistas por separado (Águila y López, 2019; Renzi 2009), sino que también existen nociones que integran dichas perspectivas.

Justamente, la complejidad de esta temática se justifica por su carácter filosófico y, en muchas perspectivas, teológico. Además, con base en la perspectiva de los derechos humanos, es fundamental en los entornos pedagógicos otorgar espacio a estas discusiones para que, por medio del pensamiento crítico, las personas puedan comprender o posicionarse sobre dichas concepciones (Ezcurdia, 1987). Así, es preciso discutir dichas perspectivas desde una posición abierta, flexible, respetuosa y tolerante. A pesar de lo anterior, sumado a los múltiples argumentos que justifican una interpretación postmoderna del cuerpo, es preciso considerar que la concepción del cuerpo vivo es posible enmarcarla en diferentes modelos antropológicos. Y, precisamente, la principal intención de superar la concepción cartesiana del cuerpo en Educación Física no es la de encajar una nueva concepción en un modelo antropológico, sino contrarrestar la desubjetivación del cuerpo (Vicente, 2010). En consecuencia, concretar «una pedagogía que trata al ser humano en toda su realidad, única y distintiva, que puede ser capaz de adentrarse en la subjetividad misma de cada persona» (Lagardera y Lavega, 2011, p. 27).

Otro argumento para considerar sobre el término Educación Física, es que el concepto educación también le otorga la esencia subjetiva a la asignatura, ya que ningún cuerpo material carente de subjetividad puede beneficiarse de un proceso pedagógico (Curcu, 2008; Ospina-Zapata, GómezVillegas y Rojas-Rojas, 2014). Esta idea toma fuerza si observamos que durante un proceso histórico progresivo, la Educación Física ha tenido diferentes transformaciones epistemológicas en función de las diferentes significaciones conceptuales que se han ido modificando. Y, justamente, una de las principales intenciones de cambio ha sido dirigida a superar la concepción cartesiana del cuerpo para integrarle a su ethos la innegable vida psíquica e intencional que lo compone (Pereira, Surdi, Tarcísio y Kunz, 2013). Cabe destacar que una de las principales líneas que ha liderado la lucha por transformar la identidad de la asignatura es la escuela de la Educación Física Crítica (Devís, 2012; Devís, Fuentes y Sparkes, 2005; Vicente, 2010). Debido al éxito gradual que aquellas transformaciones han tenido a nivel interno, es que en la actualidad es posible apreciar en torno a la literatura de la asignatura, nuevas perspectiva que abordan la justicia social, las emociones, la toma de decisiones, la motivación, las actitudes, los valores, las representaciones sociales, la inteligencia motriz y el aprendizaje motor.

A pesar de estos avances epistemológicos, es evidente que, por múltiples factores (incluso ideológicos), no todos los profesionales vinculados a la asignatura han aceptado una concepción de la Educación Física acorde a la postmodernidad, promoviendo, en ocasiones, una resistencia cultural que disminuye el ritmo de las transformaciones. Por ello, es preciso entender que el término Educación Física es polisémico, dado «que admite diversas interpretaciones, en función del contenido que se le asigne, del contexto en que se utilice o de la concepción filosófica de la persona»
(Eisenberg y Grasso, 2007, p. 63). Otro aspecto evidente y difícil de transformar, es la realidad educativa que sucede fuera de las fronteras de la Educación Física, o sea, en las otras disciplinas pedagógicas, donde el cuerpo humano se le ha otorgado un lugar segundario. Sin embargo, como ya se ha mencionado, no existe ningún acto pedagógico que excluya la corporalidad, de modo que indudablemente la idea del cuerpo vivo merece ser aplicada sin distinción de la especialidad educativa. Y, en este sentido, se ha desarrollado el concepto de corporeidad que asume dicha postura fenomenológica (Águila y López, 2019; Eisenberg y Grasso, 2007; Hurtado, 2008; Mujica, 2018b).

\section{Conclusiones y aplicaciones prácticas}

Con base en el objetivo de este ensayo, se concluye que para Husserl, Scheler y Stein, el ser humano puede ser interpretado como un ser psicofísico, un espíritu encarnado o un sujeto corporal que se expresa por medio de su cuerpo vivo y físico. Por esa razón, sus planteamientos filosóficos contribuyen a comprender que para superar la concepción mecanicista del cuerpo en la Educación Física es fundamental re-significar su concepto, dotándolo epistemológicamente de una innegable subjetividad, como se ha hecho con el término corporeidad. Por consiguiente, la terminología actual de la asignatura analizada puede ser representativa para una concepción fenomenológica del cuerpo vivo. No obstante, también se considera adecuada la posibilidad de ampliar el término de la asignatura a otros ámbitos de la asignatura que requieran ser más explicitados como, por ejemplo, el ámbito motriz.

Debido a que esta temática pertenece directamente al área de la Filosofía de la Educación Física, es que se identifican las siguientes aplicaciones prácticas: a) Re-significar desde la perspectiva fenomenológica la dimensión física del ser humano en la pedagogía y, por supuesto, en las experiencias de Educación Física que se desarrollan en esta época postmoderna; b) Superar las concepciones mecanicistas y biomédicas del cuerpo humano en torno a la Educación Física y las Ciencias de la Actividad Física y del Deporte; c) Establecer en la formación inicial del profesorado de las diferentes disciplinas pedagógicas y, en concreto, de Educación Física, cursos que aborden diferentes interpretaciones filosóficas del cuerpo humano, incluyendo por supuesto las perspectivas del cuerpo vivo de la escuela fenomenológica; d) Generar espacios de perfeccionamiento en la temática filosófica, donde el profesorado de todas las disciplinas pedagógicas pueda conocer, comprender, reflexionar y discutir las interpretaciones sobre el cuerpo humano; y e) Promover la redacción de ensayos que, refiriéndose a la temática del estudio, permitan reflexionar sobre la importancia que tiene concebir el cuerpo humano como un cuerpo vivo.

\section{Futuras líneas de investigación}

En concordancia con Águila y López (2019), se considera necesario concretar proyectos de investigación interpretativa y sociocrítica que aborden los significados, las representaciones y los imaginarios sociales en torno a la Educación Física y el cuerpo humano. Estas indagaciones 
podrían ser concretadas en todos los espacios pedagógicos pero, especialmente, en el contexto de la Educación Física por ser la disciplina pedagógica más especializada en la materia.

En concreto, en el marco de la Educación Física, se plantean las siguientes tres líneas de investigación que incluyan una discusión crítica con la perspectiva fenomenológica del cuerpo vivo y físico: a) estudios documentales que analicen las concepciones implícitas sobre el cuerpo en los currículos oficiales de la asignatura en las diferentes naciones; b) investigaciones que indaguen los significados en torno al cuerpo que han aportado diferentes referentes teóricos de la disciplina. Y, además, considerar en cada autor su contexto histórico-cultural para comprender desde una visión holística sus aportes y poder contrastarlos con una buena lectura biográfica; y c) estudios fenomenológicos que descubran las apreciaciones del profesorado y del alumnado, sobre la dimensión física del ser humano y el vínculo con su subjetividad.

\section{Referencias}

Abbagnano, N. (2008). Historia de la Filosofía. Barcelona: Hora.

Adrián, J. (2007). Hacia una fenomenología de los afectos: Heidegger y Scheler. Thémata. Revista de Filosofía, 39, 365-368.

Águila, C. \& López, J.J. (2019). Cuerpo, corporeidad y educación: una mirada reflexiva desde la Educación Física. Retos. Nuevas tendencias en Educación Física, Deportes y Recreación, 35, 413-421.

Almonacid, A. (2012). La educación física como espacio de transformación social y educativa: perspectivas desde los imaginarios sociales y la ciencia de la motricidad humana. Estudios Pedagógicos, 38 (Número especial), $177-$ 190.

Apple, M. (2011). Democratic education in neoliberal and neoconservative times. International Studies in Sociology of Education, 21(1), 21-31.

Baigorri, J., Cifuentes, L., Ortega, P., Pichel, J. \& Trapiello, V. (1995). Temas de Filosofía Cou. Madrid: Ediciones del Laberinto.

Caballero, J. (2010). Ejes transversales del pensamiento de Edith Stein. Teología y Vida, 51, 39-58.

Cagigal, J.M. (1979). Cultura intelectual y cultura física. Buenos Aires: Kapelusz.

Cagigal, J.M. (1984). ¿La Educación Física, Ciencia? Educación Física y Deporte, 6(2-3), 49-58.

Curcu, A. (2008). Sujeto, subjetividad y formación en educación para pensar en otra visión pedagógica de la evaluación. Revista de Teoría y Didáctica de las Ciencias Sociales, 13, 195-216.

Damasio, A. (1996). El error de Descartes. La razón de las emociones. Santiago: Andres Bello.

Descartes, R. (1997). Las pasiones del alma. Madrid: Tecnos.

Descartes, R. (2003). Meditaciones metafísicas. Madrid: Gredos.

Devís, J. (2012). La investigación sociocrítica en la educación física. Estudios Pedagógicos, 38 (Número e s p e cial), 125-153.
Devís, J., Fuentes, J. \& Sparkes, A. (2005). ¿Qué permanece oculto del currículum oculto? Las identidades de género y de sexualidad en la Educación Física. Revista Iberoamericana de Educación, 39, 73-90.

Díez, E. (2010). La globalización neoliberal y sus repercusiones en educación. Revista electrónica interuniversitaria de formación del profesorado, 13(2), 23-38.

Echenique, J. (2018). Dualismo socrático. Revista de Filosofía, 74, 55-72.

Eisenberg, R. y Grasso, A. (2007). Introducción conceptual a Corporeidad, Movimiento y Educación Física. En R. Eisenberg (Ed.), Corporeidad, Movimiento y Educación Física (pp. 49-90). México DF: Consejo Mexicano de Investigación Educativa.

Ezcurdia, J. (1987). Curso de derecho natural. Perspectivas iusnaturalistas de los derechos humanos. Madrid: Reus.

Fernández-Balboa, J.M. (1993). Aspecto crítico y cívico del rol de los/las profesionales de la Educación Física y el deporte. Conexiones con la política, la economía y el medio ambiente. Apunts. Educación Física y Deportes, 34, 74-82.

Ferrater, J. (2002). Diccionario de grandes filósofos 2 (K-Z). Madrid:Alianza.

Filippi, S. (2012). El alma unida al cuerpo es más semejante a Dios. Reflexiones sobre el rol de la corporeidad en la antropología tomista. Enfoques, 24, 53-62.

Freire, P. (1975). Pedagogía del Oprimido. Madrid: Siglo XXI.

Gallo, L. (2007). La educación física y su anclaje en la tradición platónico-cartesiana. Lúdica Pedagógica, 2(12), 511.

Gallo, L. (2009). El cuerpo en la educación da qué pensar: perspectivas hacia una educación corporal. Estudios Pedagógicos, 35(2), 231-242.

Gallo, L. (2012). Las practicas corporales en la educación corporal. Revista Brasileira de Ciências do Esporte, 34(4), 825-843.

Gómez, J. \& Sastre,A. (2008). En torno al concepto de cuerpo desde algunos pensadores occidentales. Hallazgos. Revista de investigaciones, 9, 119-131.

Harvey, D. (1998). La condición de la posmodernidad. Investigación sobre los orígenes del cambio cultural. Buenos Aires: Amorrortu.

Haya, F. (2003). La estructura de la persona humana según Edith Stein. En J. Sellés (Ed.), Modelos antropológicos del siglo XX (pp. 61-79). Pamplona: Universidad de Navarra.

Herrera, D. (2010). Husserl y el mundo de la vida. Franciscanum: revista de las ciencias del espíritu, 52(153), 247-274.

Hurtado, D. (2008). Corporeidad y Motricidad. Una forma de mirar los saberes del cuerpo. Educação \& Sociedade, 29(102), 119-136.

Husserl, E. (1962). Ideas relativas a una fenomenología pura y una filosofía fenomenológica. México: Fondo de Cultura Económica.

Husserl, E. (1991). La crisis de las ciencias europeas y la filosofía trascendental. Barcelona: Crítica.

Husserl, E. (1996). Meditaciones cartesianas. México: Fondo de Cultura Económica.

Lagardera, F. \& Lavega, P. (2011). Educación Física, conduc- 
tas motrices y emociones. Ethologie \& Praxeologie, $16,23-43$.

Maturana, H. (2001). Emociones y lenguaje en educación y política. Santiago: Dolmen.

Maturana, H. (2005). La objetividad. Un argumento para obligar. Santiago: Lom. ción. Barcelona: Planeta-Agostini.

Moreno, A., Campos, M. \& Almonacid, A. (2012). Las funciones de la educación física escolar: una mirada centrada en la justicia social y la reconstrucción del conocimiento. Estudios Pedagógicos, 38 (Número especial), 13-26.

Mujica, F. (2018a). Educar y suscitar emociones en la educación: Análisis crítico de su contribución al desarrollo moral. Revista de la Facultad de Educación de Albacete, 33(2), 15-27.

Mujica, F. (2018b). Las emociones en la educación física escolar. El aporte de la evaluación cualitativa. EmásF. Revista Digital de Educación Física, 51, 64-78.

Mujica, F. (2019). El dualismo cuerpo y alma en la Educación Física: análisis de las ideas de José María Cagigal. EmásF. Revista Digital de Educación Física, 60, 116-126.

Mujica, F., Orellana, N. \& Luis-Pascual, J.C. (2019). Perspectiva moral de las emociones en los contextos de educación formal. Revista Ensayos Pedagógicos, 14(1), 69-90.

Muñoz, E. (2012). Fuentes fenomenológicas de la noción de persona: su discusión en Husserl, Scheler y Heidegger. Areté, 24(1), 91-108.

Murillo, F. J. \& Román, M. (2010). Retos en la evaluación de la calidad de la educación en América Latina. Revista Iberoamericana de Educación, 53, 97-120.

Nussbaum, M. (2016). Educación para el lucro, educación para la libertad. Nómadas, 44, 13-25.

Oliva, M. \& Gascón, F. (2016). Estandarización y racionalidad política neoliberal: bases curriculares de Chile. Cadernos Cedes, 36(100), 301-318.

Ospina-Zapata, C., Gómez-Villegas, M. \& Rojas-Rojas, W. (2014). La constitución de la subjetividad en la educación contable: del proceso implícito a la visibilización de sus impactos. Cuadernos de Contabilidad, 15(37), 187211.

Pascal. (1967). Pensamientos (7ª ed.). Madrid: Espasa-Calpe. Peña, S., Toro, S., Osses, S., Pachón, J. \& HernándezMosqueira, C. (2018). Dimensión conceptual del conocimiento en Educación Física: Un estudio binacional. Re-
Merleau-Ponty, M. (1993). Fenomenología de la percep-

tos. Nuevas tendencias en Educación Física, Deportes y Recreación, 35, 170-175.

Pereira, D., Surdi, A., Tarcísio, J. y Kunz, E. (2013). Dança e expressividade: uma aproximação com a fenomenología. Movimento, 19(1), 243-263.

Platón. (2002). Fedón. Madrid: Tecnos.

Renzi, G. (2009). Educación Física y su contribución al desarrollo integral de los niños en la primera infancia. Revista Iberoamericana de Educación, 50(7), 1-14.

Rivas, A. (2015). América Latina después de PISA: lecciones aprendidas de la educación en siete países 2000 2015. Buenos Aires: CIPPEC.

Rodríguez, P. \& Hernández, F. (2018). Didáctica de la Motricidad en la formación de profesores de educación infantil. Retos. Nuevas tendencias en Educación Física, Deportes y Recreación, 34, 25-32.

Sánchez-Migallón, S. (2008). Vitalidad y espiritualidad humanas según Max Scheler. Anuario Filosófico, 41(2), 341361.

Santo Tomás de Aquino. (1993). Suma de Teología II (2ª ed.). Madrid: Bac.

Scheler, M. (2001). Ética. Nuevo ensayo de fundamentación de un personalismo ético. Madrid: Caparrós.

Scheler, M. (2000). El puesto del hombre en el cosmos. Barcelona: Alba.

Scheler, M. (2005). Esencia y formas de la simpatía. Sígueme: Salamanca.

Sérgio, M. (2006). Motricidad Humana, ¿Cuál es el futuro? Pensamiento Educativo, 38, 14-33.

Sicilia, Á. (2012). Educación Física y transformación social: implicaciones desde una epistemología posmoderna. Estudios Pedagógicos, 38(Número especial), 47-65.

Stein, E. (2004). El problema de la empatía. Madrid: Trotta.

Stein, E. (2007). La estructura de la persona humana. Madrid: Biblioteca de Autores Cristianos.

Taubenschlag, C. (2014). La noción de alma que propone Edith Stein en «La estructura de la persona humana». Revista Teología, 51(115), 69-89.

Toro, J. (2017). Educar con «co-razón». Bilbao: Desclée de Brouwer.

Vicente, M. (2010). Educación Física e ideología. Creencias pedagógicas y dominación cultural en las enseñanzas escolares del cuerpo. Retos. Nuevas tendencias en Educación Física, Deportes y Recreación, 17, 76-85.

Wesley, T. (2012). Posmodernidad y educación cristiana: desafíos ideológicos contemporáneos. Enfoques, 24(2), 85-100.
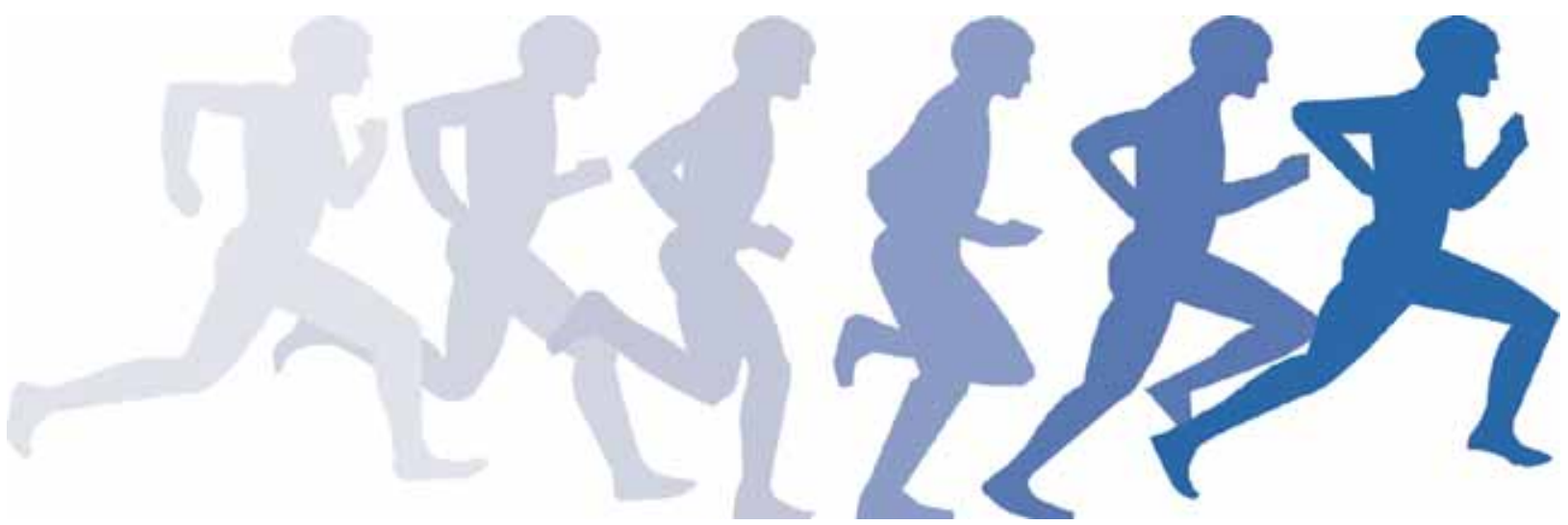